\title{
On-line monitoring of eye-position signals in contingent and noncontingent paradigms*
}

\section{STEPHEN M. REDER \\ The Rockefeller Lniversity. . Vew York. Vew York 10021}

This paper presents a research methodology for the study of human attentional and perceptual processes by means of on-line monitoring of eye-position signals. The first part of the presentation considers techniques by which commercially available apparatus may be interfaced to a minicomputer for purposes of monitoring eye-position signals during psychological experiments. Hardware and software techniques related to automating the calibration, recording, and analysis of eye-position data are discussed. The second part of the paper discusses a more advanced research methodology, one in which visual stimuli are contingent upon momentary eye-position signals. The methodology is appropriate to a variety of studies in which eye movements are considered to be part of an attentional control system. Several experimental applications are described. The implementation of eye-position-stimulus contingencies on a small computer poses additional technical problems, several of which are discussed.

This paper discusses a minicomputer system for monitoring human eye position and eye movements during psychological experiments. The aim of the paper is to acquaint researchers using computer-based laboratories with some of the hardware and software considerations for routinely incorporating eyc-position signals (hereafter abbreviated EPS) as dependent variables in a variety of psychological experiments, and in some cases, to use the EPS actively for process control of computer-based experiments.

The paper is divided into two major sections. The first section discusses techniques for automating the measurement, calibration, recording, and analysis of EPS, considering requirements of both hardware and software. The second section introduces a powerful research methodology appropriate to several types of experimental investigations. In this methodology, the choice of visual stimuli presented to a $S$, and/or the manner in which they are presented, is programmed to depend upon certain characteristics of the S's momentary EPS. Such experimental procedures will be termed contingent paradigms, as distinguished from noncontingent paradigms in which EPS are recorded on-line but are not used to control the experiment. The research potential of contingent paradigms is illustrated by several experimental applications.

The use of EPS in experimental psychology has a long history (Kling \& Riggs. 1971), and includes applications

\footnotetext{
*This research was supported in part by Grant GM 16735 from the National Institute for General Medical Sciences and by Grant TO1 GM 01789 from the U.S. Public Health Service to The Rock efeller U niversity.
}

to numerous research topics. For the present, however two categories of applications are of interest (1) applications which use the sequence and timing of the fixation points of the eyes as indicators of attentional processes operating during visual perceptior (e.g.. Loftus ${ }^{1}$ ), during learming (e.g.. McC ormack. 1970) and during other cognitive activities (Mackworth $\&$ Bruner. 1970): and (2) use of EPS data for physiological or psychophysical studies of the visual ststem as it interacts with the eve-movement svstems (Yarbus. 1967 Latour. 1966).

\section{CONSIDERATIONS FOR NONCONTINGENT PARADIGMS}

The discussion below is pertinent to the implementation of an on-line system for noncontingent paradigms. Additional technical considerations pertinent to implementing a system for use in contingent paradigms are discussed in later sections.

Measurement Techniques

Several techniques have been used to generate EPS from human Ss (Yarbus, 1967: Kling \& Riggs, 1971). For general-purpose use in a psychological laboratory: the technique of ocular reflectance (Smith \& Warter, 1960; Rashibass, 1960) often provides a satisfactory compromise between the opposing requirements of measurement accuracy and overall ease of use in a variety of experimental settings. In the infrared reflectance techniques. the surface of the S's eye(s) is illuminated with low-intensity infrared light. The IR reflected off the cornea can be analyzed by several analog methods. By exploiting differences in the reflectance properties of iris, pupil, and sclera, signals from IR-sensitive photoconductors can be processed electronically to yield $\mathrm{dc}$ outputs proportional to the angular displacement of the eye(s) from the position of primary gaze. Apparatus are available commercially ${ }^{2}$ or can be built inexpensively for analyzing the IR reflection patterns, which, when properly implemented, emit accurate $^{3}$ data about the position of the eyes relative to a fixed orientation of the head ${ }^{4}$; hence, in most experimental settings, the S's head should be firmly fixed, most successfully accomplished by means of a biteboard. ${ }^{5}$

\section{On-Line Measurement}

There are several advantages to recording EPS on-line. as opposed to procedures which record eye position photographically (cf. Mackworth \& Mackworth. 1958) or on XY-plot recorders. Given the availability of a minicomputer laboratory. the on-line measurement and 


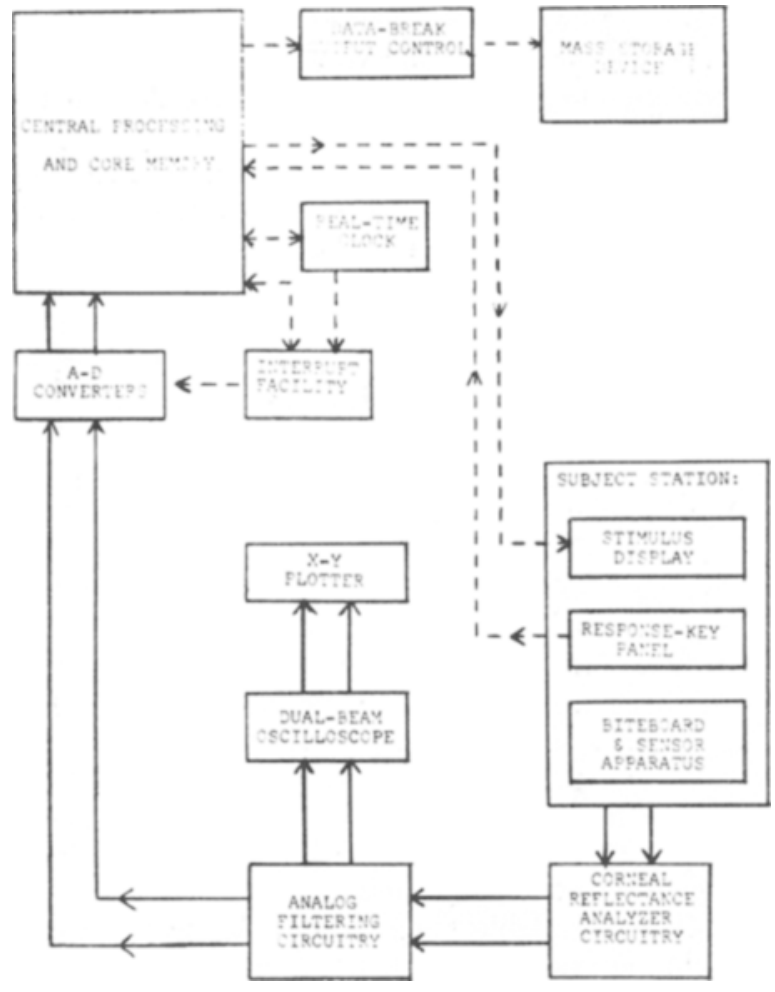

Fig. 1. Hardware components of on-line eye-position monitoring system. The parallel solid lines show the transduction path for the eye-position signals. The broken lines show the digital lines of communication between the system components. In our laboratory, the central processor is an 8K PDP-12, equipped with an AD12 analog-to-digital converter option, a $\mathrm{KW}-12 \mathrm{~A}$ real-time clock option, and using a TC12 data-break LNCtape control for mass storage. The stimulus display system is an auxiliary VR-12 display terminal. The sensor apparatus and ocular reflectance analyzer are modified from Biometrics Model SGH-V2.

recording is typically easier to calibrate and less expensive than recording on film or heat-sensitive paper. Considerable effort is saved in data analysis when the EPS are recorded digitally, as the on-line records can be readily processed under program control, bypassing the painstaking, tedious process of digitizing film or paper records by hand (cf. Loftus, 1972). ${ }^{6}$ The most important advantage, however, of on-line monitoring is the opportunity it provides for using the contingent paradigms considered in the second section of the paper.

\section{Hardware Considerations - for Noncontingent Paradigms}

The basic hardware environment required for on-line monitoring of EPS in noncontingent situations is diagrammed in Fig. 1. An ocular reflectance apparatus and biteboard setup is located at the $S$ station along with other experimental apparatus (e.g.. CRT display for presenting stimuli. response panels. ctc.). Figure 2 is a close-up photograph of an experimental station used in our laboratory. The infrared sources and photocell assemblies are modified from a Biometrics apparatus and are attached by an XYZ movement to the biteboard holder. ${ }^{7}$ It is possible to attach the IR sources and photocell assemblies to spectacle frames rather than to the biteboard holder directly. If the $S$ is equipped with a tight-fitting biteboard, ${ }^{8}$ however, there is typically less movement of the sensor assembly with respect to the head when they are mounted on the biteboard holder directly. In addition, whenever Ss are to be run in several experimental sessions, there are additional advantages to having the sensor assembly attached to the biteboard holder rather than on spectacle frames.

Once a biteboard is fitted for a $S$ and the positions of the sensors are suitably adjusted by the $\mathrm{XYZ}$ movement, the spatial coordinates of the positions relative to the biteboard are noted. These positional adjustments need be made only once for a given $\mathrm{S}$, as they can be rapidly reproduced in subsequent sessions by resetting the recorded $\mathrm{XYZ}$ coordinates.

Signals generated by the ocular reflectance apparatus are fed through appropriate analog circuitry and then are amplified for input into the computer through analog-to-digital conversion channels. The computer samples these dc signals at a programmed rate by means of a real-time clock. This is most easily accomplished by use of a variable-frequency crystal oscillator clock which can be tied to the program-interrupt facility of the computer. The sampled signals (after suitable digital processing) are recorded on a mass-storage device by means of rapid output-buffering software (described below). If a magnetic tape unit is used for mass storage, it is essential that the tape-control and central processor hardware be such that signals can be sampled, processed,

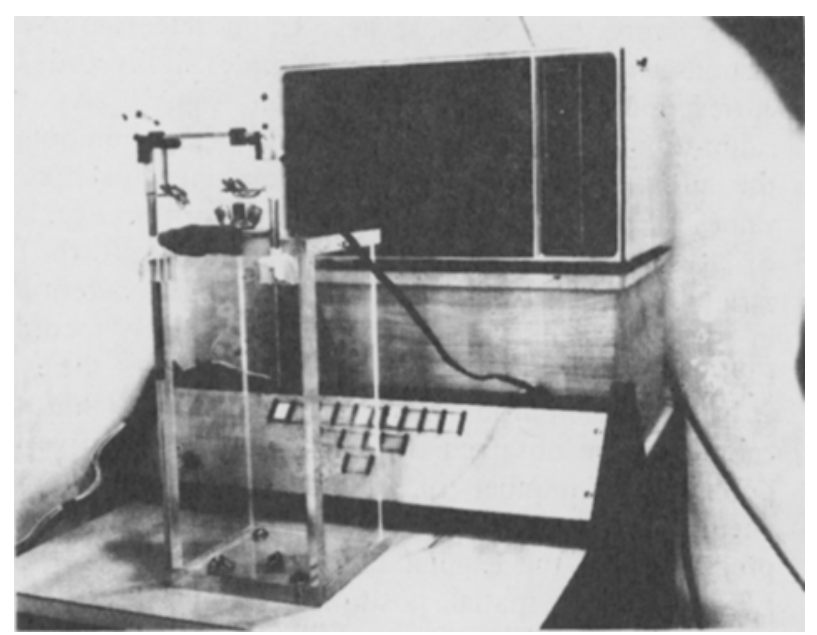

Fig. 2. Photograph of S station. The VR-12 display terminal, the manual response-key panel. and the combination biteboard-sensor apparatus are on a long table in an adjoining room to the computer. The $S$ is seated at the end of the table nearest the biteboard apparatus. facing the response pand and display terminal. 
and stored in one area of core memory while previously stored signals are simultaneously output from a different part of memory. ${ }^{9}$ If such hardware is not available. there will be inevitable periods of "dead time" (during which the EPS cannot be sampled) whenever an output operation to the mass-storage device is in progress.

The oscillographic plotter and oscilloscope shown in Fig. 1 are not essential components of the on-line system, but prove quite useful for troubleshooting occasional apparatus failures or occasional difficulties in sensor adjustment or calibration.

\section{Software Considerations \\ for the Noncontingent System}

The routines described below typically occupy small amounts of memory (1,000-2,000 words on a 12-bit machine) when programmed efficiently in Assembly language. Once written and debugged, they can be routinely incorporated into a wide variety of experimental programs as software modules. Additional software considerations appropriate to contingent systems are discussed in later sections.

\section{Calibration}

The aim of the calibration routine is to sample and record EPS values which correspond to fixations of the eye on a specified set of points of known angular displacements from the position of primary gaze. Subsequently recorded experimental EPS can be converted into spatial positions of the eyes through reference to the matrix of calibration values by means of suitable interpolation procedures.

One convenient means of obtaining the calibration values is to have the $S$ successively fixate each point of the grid of calibration points. While fixating each point, the $S$ depresses a response key. Upon detection of the response, the calibration routine samples and records the corresponding $\mathrm{X}$ - and $\mathrm{Y}$-position signals. As the calibration proceeds through the grid of fixation points, the program should check the obtained calibration values in terms of desired requirements for linearity, lack of cross-talk between $X$ and $Y$ signals, and so forth. The strictness of the calibration criteria imposed determines in part the accuracy with which subsequently recorded EPS data can be assigned to spatial positions of the eyes.

For general-purpose use, it is suggested that the calibration be obtained over an $m$ by $n$ grid of fixation points. The number of calibration points and their angular separations will depend on the degree of precision and the angular range desired for converting EPS data into spatial positions. Other factors being equal, the more linear the EPS response to angular displacement of the eyes, the fewer calibration points are needed to achieve a given level of precision.

It is desirable to conduct the calibration under stimulus conditions which closely resemble those in which the experimental EPS will be subsequently recorded. For example. if EPS data were to be recorded while a $S$ read textual material. it would be advisahle to superimpose the calibration points on a stimulus background which was similar in visual format whe experimental text materials ( see Fig. 63).

It is advisable to carry out a calibration cach time the $S$ assumes a position in the biteboard. as slight changes in position of the head relative to the sensor apparatus can alter EPS values. Ss in our laboratory typically remain in the biteboard apparatus for 10-min blocks of experimental trials, with a brief rest period and $30-5 e c$ calibration preceding each block. To verify the validity of the initial calibration for the entire block, a calibration may be taken at the end of the block and compared with the initial calibration.

\section{EPS Sampling}

The rate at which experimental EPS are sampled should depend in part on the desired precision for resolving the temporal boundaries of intervals in which the eye is stationary (i.e.. fixating) and moving. Often the maximum sampling rate is determined by the temporal characteristics of the EPS-generating apparatus and/or the analog filters and amplifiers which deliver the signals to the A-D channels.

Once a suitable sampling rate has been selected. the real-time clock should be programmed to generate program interrupts at the desired rate. The interrupt-handling software should sample and save the momentary $\mathrm{X}$ - and $\mathrm{Y}$-position voltages and perform any additional signal processing before returning control to the mainline experimental program.

\section{Real-Time EPS Recording}

In the simplest situation, the sampling routine described above returns only the $\mathrm{X}$ - and $\mathrm{Y}$-digitized voltages to the mainline program for storage. These values can be recorded sequentially on a suitable mass-storage device to form a real-time record of eye position. The creation of a real-time record is most easily implemented by means of a buffered-output subroutine, $\operatorname{RECORD}(X)$, which enters the parameter $X$ into the sequential real-time record.

Figure 3 diagrams the structure of RECORD. Associated with this subroutine is a memory storage array of $2 \mathrm{~N}$ words, where $\mathrm{N}$ is the number of words per physical block of the mass-storage device. When either half of this $2 \mathrm{~N}$-word array has been filled, it is output into a block of the mass-storage device, while the central processor remains free to continue sampling, processing, and storing subsequent signals in the remaining $\mathrm{N}$ words of the array. 10

The RECORD subroutine may also be used to enter other variables into the real-time record, such as occurrences of experimental events. S's responses, calibration values, trial-identification words, and so forth.

The advantages of recording in real-time mode (as compared to the compressed-data mode described 


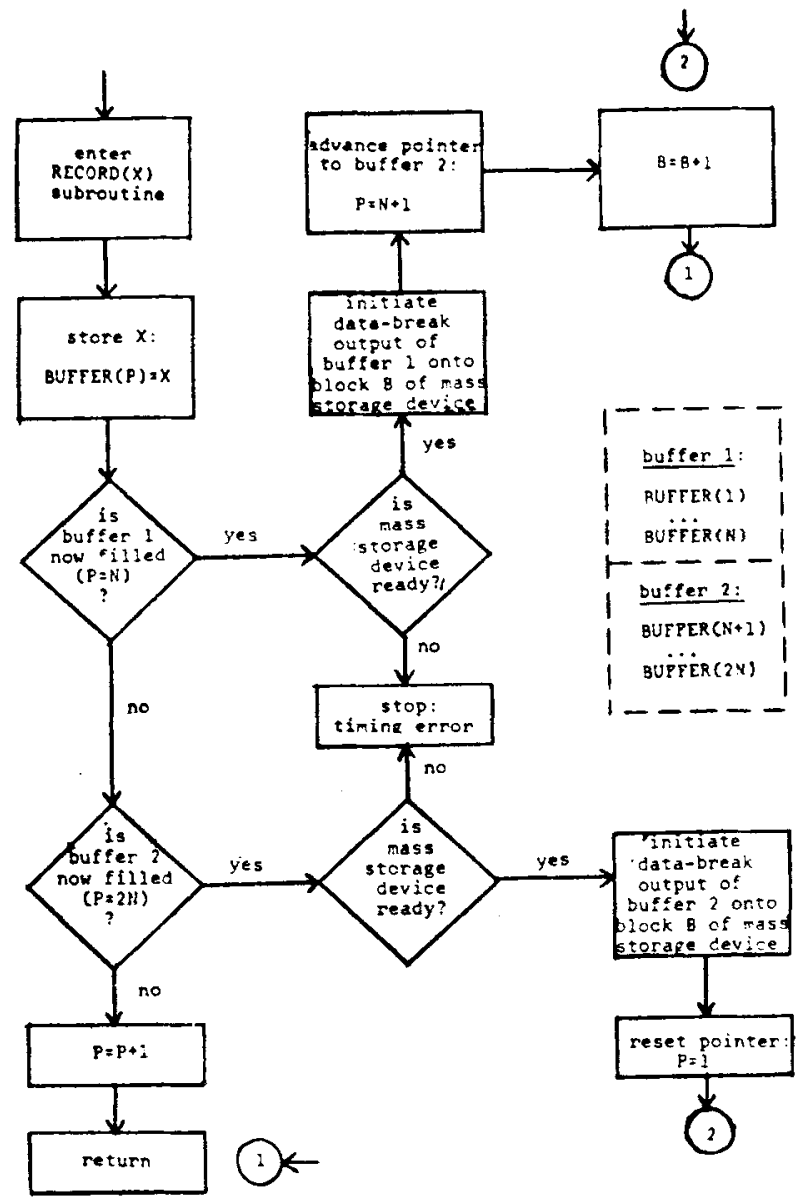

Fig. 3. Flow chart for the RECORD(X) subroutine. $X$ is the parameter of RECORD to be stored. $N$ is number of words per physical block of storage on the mass storage device. $P$ is a pointer to the next word in the memory buffer array. $B$ is the number of the next available physical block on the mass-storage device. The memory buffer array, BUFFER(), is indicated in broken lines and contains $2 \mathrm{~N}$ words, broken into two halves. See text for explanation.

below) are (1) the small amount of software needed, and (2) the flexibility available for postexperimental data analysis of the real-time record. On the other hand, vast amounts of auxiliary storage are required for the real-time records. For example, if two signal values (an $X$ and a $Y$ ) are sampled 60 times per second, then a minimum of 7,200 words per real-time minute will be stored. In many situations, the excessive storage requirements for real-time records are not compensated for by the advantages mentioned above. When such is the case, an alternative recording format is possible. Such formats compress the real-time data into a more economical form.

\section{Compressed EPS Recording}

In order to economize on auxiliary storage, software analysis of the EPS signals may be performed on-line between successive samples. The analysis module is called by the interrupt handler after each EPS sampling operation is completed. The goal of this software module is to compress the real-time sequence of EPS values into a sequence of fixation and eye-movement data.

One possible software compression routine, COMPRESS, is described below. It has proved satisfactory for processing EPS data in situations where the data are to be analyzed only in terms of the spatial location and duration of each fixation of the eyes, and the duration of saccadic eye movements between the successive fixations. Different software is required for situations in which smooth pursuit movements of the eyes occur during tracking tasks (Robinson, 1965) or in situations where data are desired on vergence adjustments of the eyes (i.e., where independent $\mathrm{X}$-position signals are obtained from each eye).

The COMPRESS routine resolves the real-time EPS sequence into the following type of data sequence:

$$
\ldots, P_{n}, D_{n}, S_{n}, P_{n+1}, D_{n+1}, S_{n+1}, \ldots
$$

$P_{n}$ is the location of the $n^{\text {th }}$ sequential point of fixation; the location may be specified by the raw EPS voltages 11 (to be converted into spatial locations by subsequent reference to the calibration matrix) or as $X$ and $Y$ coordinates, derived on-line by suitable comparison and interpolation from the calibration matrix. $D_{n}$ is the duration of the $n^{\text {th }}$ fixation (measured readily in units of the number of real-time sampling periods). $S_{n}$ is the duration of the saccadic eye movement following the $\mathrm{n}^{\text {th }}$ fixation interval and preceding the $\left.\mathrm{n}+1\right)^{\text {th }}$ fixation interval, also measured in units of real-time sampling periods.

This compressed data sequence may be buffered onto the mass-storage device by the RECORD subroutine described earlier. The logical structure of the COMPRESS module is diagrammed in Fig. 4. The routine assumes that at any given point in time, the eyes are either stationary (i.e., in a state of fixation) or moving (i.e., a saccade is in progress), represented by the program states of FIXING and MOVING, respectively. Whenever a new fixation is detected, state FIXING is entered, the fixation timer is initialized, and a subroutine THRESH is called, which computes suitable velocity thresholds for subsequent detection of a saccade onset; this computation is made through reference to the calibration matrix ${ }^{2}$ and is based on velocity characteristic of the saccades (Yarbus, 1967; Latour, 1966). Each time the EPS are sampled, a subroutine, SPEED. is called which returns an estimate of the momentary velocity of the eyes. ${ }^{13}$ Each time the momentary velocity estimate falls below the velocity threshold computed by THRESH, the fixation is judged to persist. the fixation timer is incremented, and the FIXING state is maintained. When a superthreshold velocity is estimated, the fixation is judged to have ended. and a transition is made to the state MOVING. after the position and duration information $\left(P_{n}\right.$ and $\left.D_{n}\right)$ for the preceding fixation have been stored 
Fig. 4. Flow chart for software of COMPRESS module. Logical structure of an example of software which resolves and stores successive eye-position samples into a sequence of fixations and saccades. STATE assumes the value 0 whenever a fixation is in progress and the value 1 whenever a saccadic eye movement is in progress. $V$ is the magnitude of the estimated instantaneous velocity (computed by subroutine SPEED). $D$ and $S$ are counters which time (in units of number of samples) fixations and saccades, respectively. $T_{f}$ and $T_{s}$ are the magnitudes of the threshold velocities for onset and offset of a saccade, computed by the subroutine THRESH. Four exits from the module are distinguished for use in contingent situations (described in later sections of text). See text for explanation.

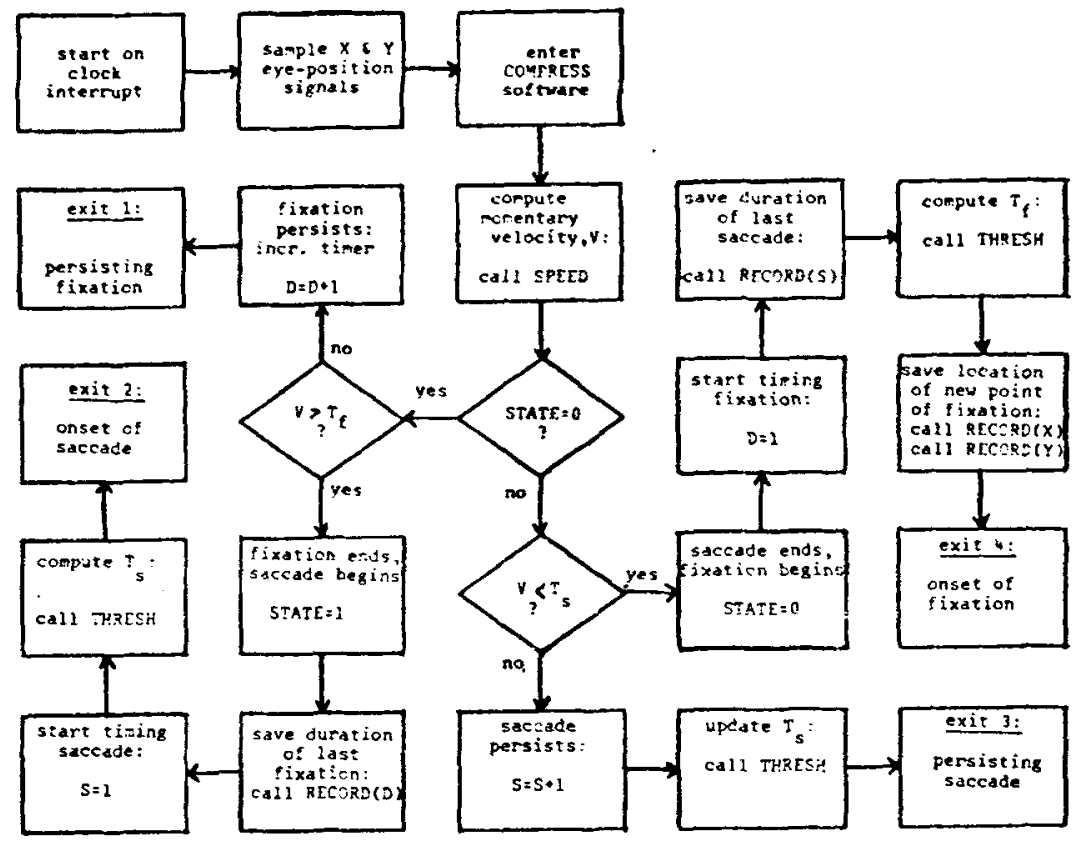

When state MOVING is entered, the saccade timer is initialized. In MOVING, the situation is reversed: the saccade is judged to persist as long as momentary velocity estimates stay above threshold. Each time the EPS are sampled from the MOVING state, the saccade timer is incremented, and a velocity estimate is obtained by calling SPEED. Unlike in the case for the FIXING state, however, the threshold velocity must be updated with each sample (unless the EPS are linear over the range of the saccade). The FIXING state is reentered when the momentary velocity estimate is subthreshold, at which time the saccade duration $S_{n}$ is entered into storage.

Before the COMPRESS module (or other related software) is implemented into the on-line system, it is advisable to check the accuracy with which it discriminates the fixation and saccade intervals. This can be checked readily by obtaining a real-time EPS record, and then analyzing the record with a data analysis routine identical to the particular COMPRESS module to be used. Comparison of the real-time and compressed-data records will reveal any systematic errors in the software.

\section{Comments on a Few Technical Problems 120-Hz Noise in EPS Signals}

Many ac-supplied light sources emit radiation which interferes with the IR ocular reflectance apparatus. The presence of such interference appears when the EPS are input into ac-triggered oscilloscopes, showing up as a narrow band of noise centered at $120 \mathrm{~Hz}$.

Although suitable electronic filtering can suppress these ac artifacts, such filters typically distort genuine signal components near the $120 \mathrm{~Hz}$ band. This distortion selectively damps the low-velocity components of the saccades (occurring near the fixation-saccade boundaries), making more difficult the discrimination of the fixation-saccade boundaries by the COMPRESS software. Furthermore, such filtering induces an inevitable delay of $15-20 \mathrm{msec}$ in the arrival of the signals to the A.D conversion channels, a delay which proves to be serious in the contingent paradigms.

If these distortions are not acceptable, one of two alternative remedies for the ac artifacts are suggested: (1) Use dc-supplied lighting for ambient illumination and for the apparatus which present visual stimuli to the $S$. (2) If the use of dc-supplied apparatus is not feasible (as is often the case for many stimulus displays), all EPS sampling (including calibration) should be rigidly phase-locked to the ac power source. The ac artifacts can be eliminated by digitally filtering the signals by sampling at $120 \mathrm{~Hz}$ or some multiple thereof.

\section{Blinking Artifacts}

When it is essential to distinguish blinks of the eye in the data record, the calibration should include several brief EPS records sampled while the $S$ intentionally blinks. For example, the $S$ could press a response key before and after he deliberately blinks; in practice, this "blink" record can be referenced to distinguish blinks in the experimental record.

\section{Convergence-Divergence Adjustments}

To identify the vergence adjustments which accompany fixation changes (Yarbus, 1967), it is necessary to calibrate and measure EPS (in the horizontal meridian) from both eyes simultaneously. In many contingent situations where advance knowledge of an impending saccade is desirable, the detection of vergence adjustments can be quite useful, as they tend to begin in advance of the onset of the saccadic eye movement (Yarbus, 1967). 


\section{Distinguishing Translational Shifts from Eye Rotations}

Most ocular reflectance techniques fail to distinguish translational shifts of the globe within the orbit from binafide rotations of the eye. More sophisticated measurement techniques are available to effect this distinction. such as those used by Cornsweet (1958) or Rashbass and Westheimer (1961).

\section{CONSIDERATIONS OF METHODOLOGY AND IMPLEMENTATION FOR CONTINGENT PARADIGMS}

As mentioned above, the term "contingent paradigm" refers to an experimental situation in which the choice of visual stimuli presented to a $S$ and/or the manner in which they are presented depend upon certain characteristics of the EPS record. Under consideration here are only those situations in which the contingencies refer to characteristics of the momentary EPS, situations in which the on-line system must react extremely rapidly to a change in eye position or state (e.g., from fixation to saccade). Paradigms in which the contingencies are more remote, such as where the independent variables for Trial $n+1$ depend on EPS data from Trial $n$, will not be considered; the noncontingent techniques previously described are appropriate to these situations.

Several simple contingent paradigms have been used in perceptual research, most notably in investigations of visual thresholds during saccades (cf. Latour, 1962; Volkmann, Schiff, \& Riggs, 1968) and of stimulus control of the smooth pursuit movements of the eyes during tracking tasks (e.g., Rashbass \& Westheimer, 1961; Yarbus, 1967). However, the techniques used were specialized to the requirements of the particular experiments and were not implemented through the use of an on-line computer system. It is therefore the purpose of this section to suggest the usefulness of contingent methodologies for a broader class of applications and to consider generally applicable techniques for their implementation in minicomputer systems.

\section{Simple Contingencies}

In the simplest contingent situations, the momentary EPS monitored on-line can be used to maintain a specified relation between visual stimuli and the S's eye position. A variety of contingencies may be imposed, including ones which maintain a specified spatial relation between fixation point and stimulus and ones which maintain a specified temporal relation between stimulus onset and onset of fixation (or eye movement). An example of the use of these simple contingencies in our laboratory is given below.

Example $A$-Time course of perceptual processing during a fixation.

It has been suggested (cf. Latour. 1966) that visual processing during a fixation interval is time-locked to eye movements preceding the fixation. Several experiments test the validity of this hypothesis in standard experimental tasks used to study visual information processing (Haber, 1969). In these situations, the $S$, at his own pace, initiates the stimulus display by executing a prescribed saccadic eye movement from cne point to another. The stimulus is then exposed briefly in a specified spatial position relative to the fixation point established by the initiating saccade. The onset of the stimulus is a contingent variable, timed to occur at a specified temporal lag relative to the onset of fixation. The stimuli are multisymbol arrays, the details of which vary according to the task. In all tasks, the $S$ is required to respond rapidly and accurately by pressing one of two alternative response keys.

The tasks include standard two-alternative detection tasks (Estes \& Taylor, 1964), same-different tasks (Egeth, 1966), and set-membership tasks (Atkinson, Holmgren. \& Juola, 1969). In addition to the usual independent variables employed in these tasks, the contingent variables of temporal and spatial position of the stimulus with respect to fixation (as described above) are manipulated experimentally, as well as the size and direction of the initiating saccade.

\section{Methodologies for More Complex Contingencies}

Experimental contingencies of a more complex nature may be implemented in mini on-line systems. The use of these contingencies is often appropriate when the behavior under study is modeled in terms of a control system theory for eye movements. In the framework of such a model. visual information processed from the fixation intervals generates feedback to the perceptual (or attentional) control system. The successive fixations made by the eyes are discrete sampling operations directed by the control system. In some models, the goal of the control system is to predict various characteristics of the visual stimulus: the choice of fixation points reflects selective sampling of those parts of the stimulus judged most pertinent to the verification or updating of the system's predictions. These predictions are based in part on feedback from preceding sampling and in part on information generated by cognitive and sensory sources.

The gross structure of a control system model for saccadic eye movements is diagrammed in Fig. 5. The details of the model will depend, of course, on the particular behavior being studied with the aid of eye-position monitoring. In many situations, suitable contingent paradigms may be powerful experimental tools for determining the detailed manner in which the control system operates. Contingent manipulations designed to interfere with selective aspects of the feedback signals or the sampling operations often will reveal the structure of the model control system and the nature of the information on which it depends. The example below illustrates the use of a contingent methodology more complex than that described in Example A. 
Example B-Visual search with controlled sampling and feedback.

In visual search tasks requiring voluntary changes of fixation point (e.g.. Neisser. 1964), issues often arise concerning the amount of information sampled during each fixation and the extent to which the choice of successive fixation points depends on peripheral vision. In terms of a control system model for visual search. these issues can be investigated by comparing visual search under various conditions in which the $E$ controls the amount of information available for sampling during each fixation as well as the availability of peripheral cues (as feedback) to the system controlling the selection of fixation points. These controls can be imposed by use of a contingent paradigm.

In our laboratory, a contingent situation has been set up in which only a small region of the stimulus array being searched is displayed clearly during any fixation. By using a computer-controlled CRT display, only that part of the stimulus array within a specified region around the momentary point of fixation is displayed clearly: outside of this region, only certain specified features of the stimulus are displayed, thus varying the richness of the peripheral cues available for selecting subsequent fixation points. The controlled areas of clear central vision and degraded peripheral vision follow the viewer as he scans the array with his successive fixations.

The final example to be presented of contingent paradigms is taken from experiments being conducted in our laboratory on the perceptual control of reading. The methodology of these experiments is closely related to the predictive control system model outlined above.

Example $C$-Reading with controlled domains of perception.

The reader is modeled as an information processor whose successive fixations sample information from the text. As he reads, he attempts to predict certain features of the text (and of the message contained therein). These predictions are based in part on information acquired from previous fixations, in part on knowledge of the style and content of the material, and in part on linguistic considerations (cf. Hochberg, 1970). The selection of subsequent points of fixation is controlled in a manner which permits the verification and updating of the control system's predictions.

From the perspective of this model, several crucial theoretical issues arise which are readily explored with a contingent methodology. Several examples of these issues, related to those discussed above under Example B, concern (1) the size of the text area sampled in each fixation. (2) the area of text peripheral to the fixation point from which peripheral cues are processed. and (3) the question: upon which features of the peripheral text does efficient selection of fixation points depend?

The $S$ is seated at an experimental station (see Fig. 2) and assumes a position in the biteboard apparatus facing a CRT display. A brief calibration period precedes each

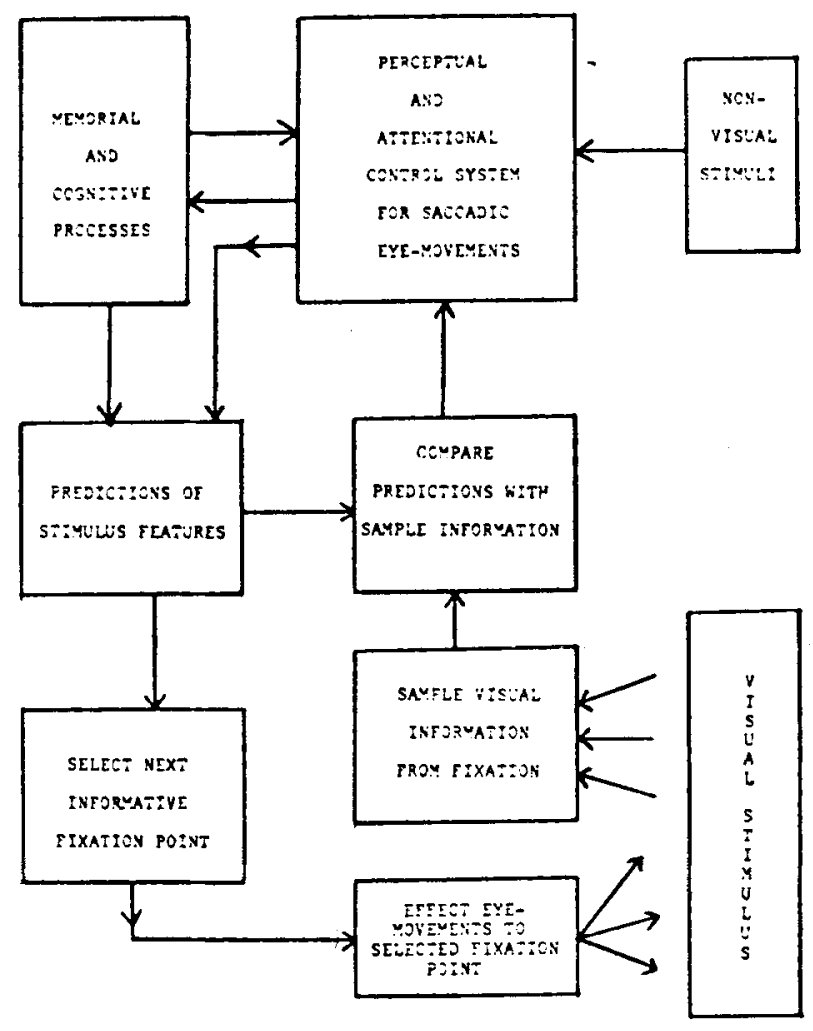

Fig. 5. Gross structure of a predictive control system model for saccadic eye movements. See text for explanation.

experimental run. During calibration. a page of nonreadabie text-iike characters is dispiayed, and an " $\mathrm{X}$ " is superimposed on the page successively at each point of the calibration grid. Figure 7a shows one such point superimposed during a calibration. Following the end of calibration, the $S$ reads successive pages of text displayed on the CRT. As the S scans the page by making saccades, the text display is contingent upon the location of each point of fixation. The basic contingencies are imposed by displaying a simulated "fovea" and simulated "periphery" around each fixation point. All characters of the text which fall within a momentary "fovea" are displayed clearly, while all characters of the text outside of the "fovea" (i.e., in the simulated "periphery") are displayed in a degraded manner, with only certain specified features displayed. The size of the simulated "fovea," its relative position to each fixation point, and the nature of the information available in the simulated "periphery" (e.g., blanks between words, punctuation marks, etc.) serve as independent variables. Generally, the values assigned to these variables will change with each new page of the passage (pages are "turned" by the depression of a response key), giving within-S, within-passage comparisons of reading under the various conditions. Figures $6 \mathrm{~b}-\mathrm{d}$ and 7 are photographs of displays made under some of these conditions. At the end of the passage, a multiple-choice comprehension test is administered on the CRT display. 


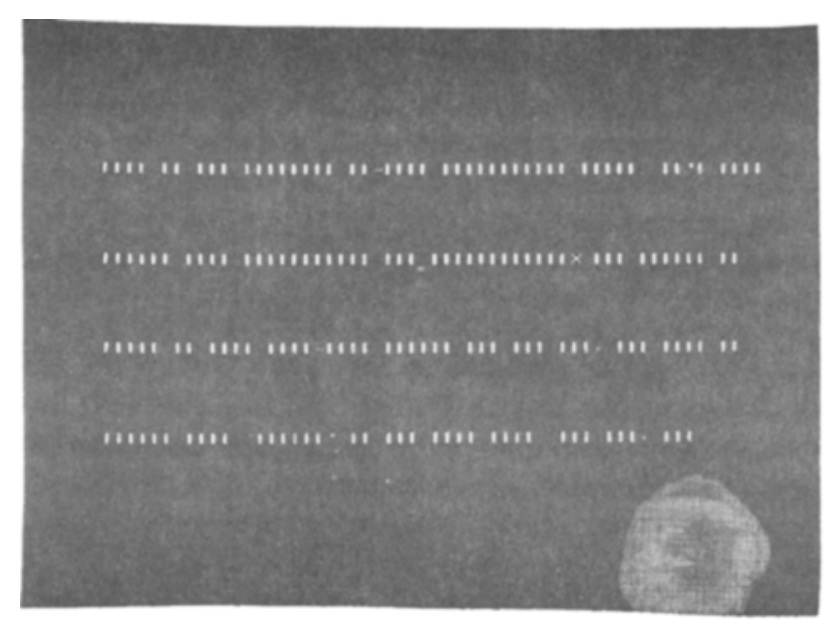

Fig. 6 (a)

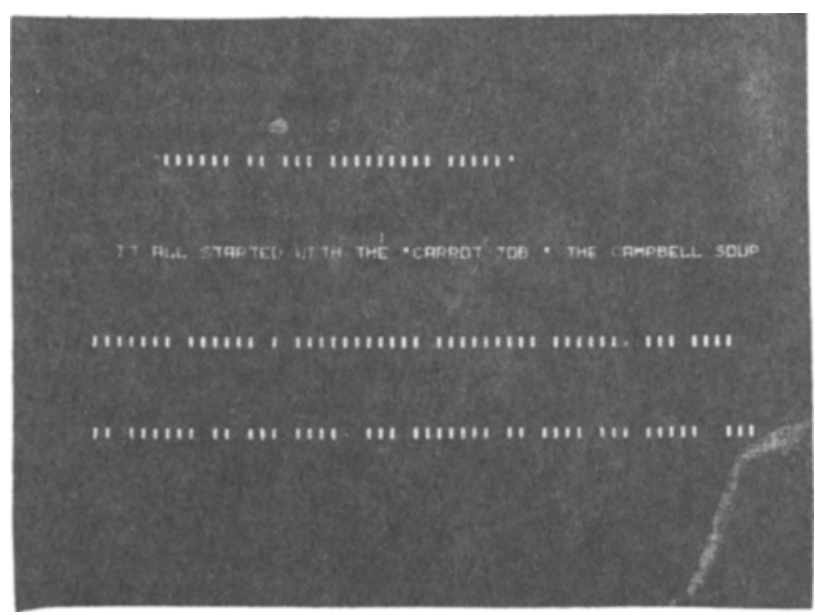

Fig. 6 (b)

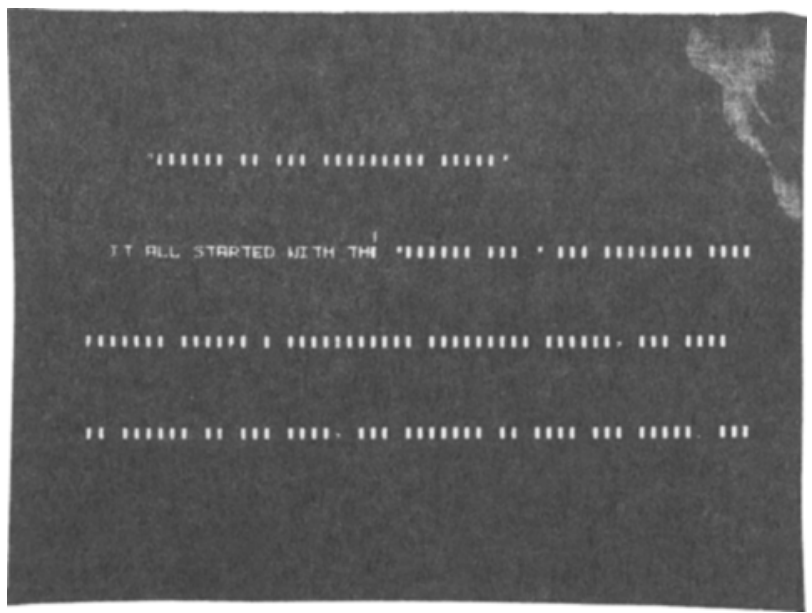

Fig. 6 (c)

Fig. 6. Photographs of example displays presented in contingent reading experiments. (a) Background dummy-text stimulus used during calibration. Note the superimposed " $\mathrm{X}$ " on
Fig. 6 (d)

which the $S$ fixates for calibration. The calibration is obtained over a grid of Xs superimposed one by one over the background stimulus. (b-d) Typical displays of text contingent on position of fixation. The cursor marks the point of fixation (not seen by the $S)$. In each case, a region of the text around the fixation point is displayed (simulated "fovea") clearly, while the remaining areas of text are displayed as a simulated "periphery," in which the positions of letters are displayed (but not their identities) and blanks and punctuation marks are displayed. (b) Entire line being fixated is "foveal." (c) All text positions on fixated line to left of fixation point are "foveal." (d) All text positions on fixated line to right of fixation point plus five to left are "foveal."

The hardware and software components of noncontingent EPS-monitoring systems were described above. The implementation of contingent paradigms generally requires additional hardware components and often imposes severe constraints on software programming. The following considerations may prove helpful for implementation in a minicomputer-based laboratory.

Hardware Considerations for the Contingent System Computer-Controlled Stimulus Display

In addition to the previously mentioned hardware components for the noncontingent situations, appropriate apparatus for displaying stimuli under computer control is required. The most convenient means for presenting computer-controlled visual stimuli is some type of CRT display interfaced to the computer. If multisymbol displays are to be used, it is advisable to utilize a display system that uses hardware character generation.

In many contingent situations, the stimulus presented to the $\mathrm{S}$ often must be changed or updated whenever a change of fixation point occurs. The latency of the display change with respect to the detection of the onset of a fixation (or eve movement) depends upon hardware characteristics of the display system. The principle feature of interest is the rate at which the display can be 


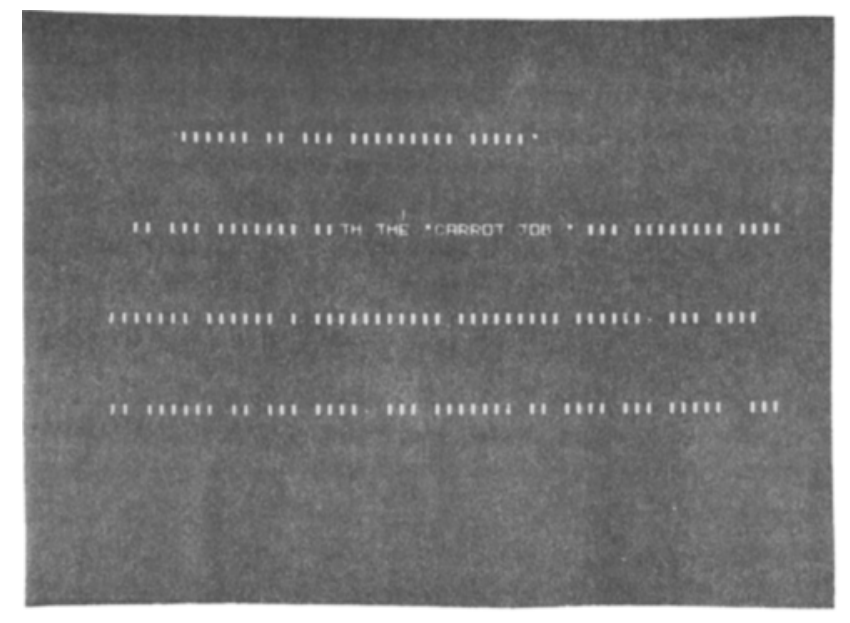

Fig. 7 (a)

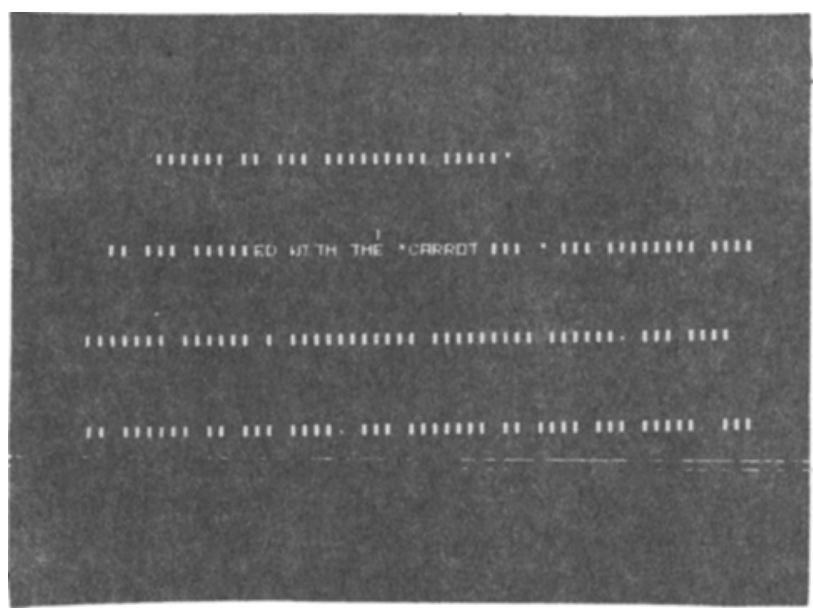

Fig. 7 (b)

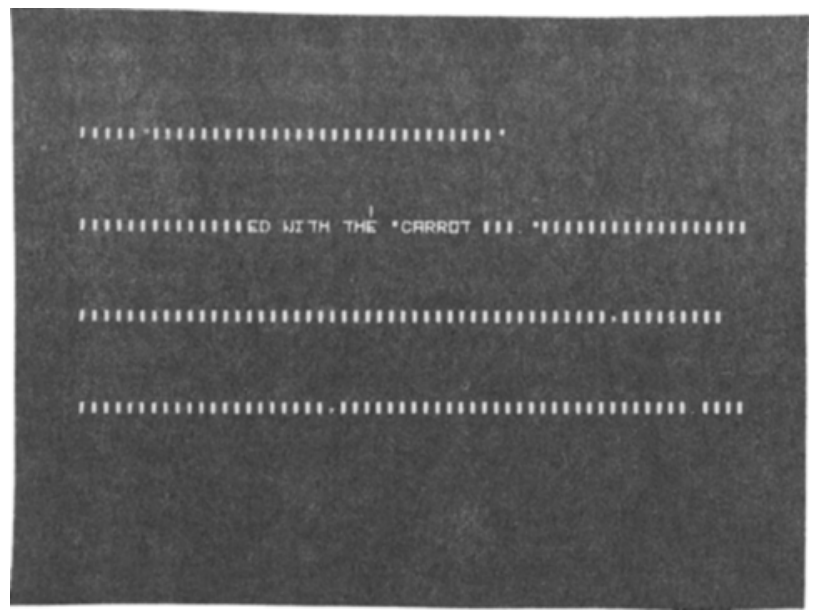

Fig. 7 (c)

Fig. 7. Additional photographs of example displays presented in contingent reading experiments. Width of simulated "fovea" is 20 characters. (a) "Fovea" is centered five characters right of

\section{H11,}

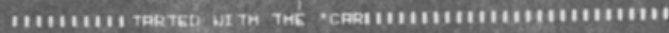

Bu+

(1)

Fig. 7 (d)

fixation point; "periphery" as in Fig. 6. (b) "Fovea" is centered on fixation point; "periphery" as in Fig. 6. (c) "Fovea" is centered on fixation point; "periphery" as in Fig. 6, except no information about interword spaces is available. (d) "Fovea" is centered five characters left of fixation point; "periphery" contains no information about interword spaces or punctuation marks.

changed. The fastest input rates to character-display systems are typically associated with nonstorage-type CRT displays (such as the VR-12 display interfaced to a PDP-12 in our laboratory). Although such display systems are well suited for rapid stimulus updating. 14 two drawbacks to the use of such displays are (1) the substantial amounts of central processing time required for refreshing large stimulus arrays, a requirement which often constrains the amount of time available for EPS sampling and processing software, and (2) the size of the stimuli displayed is often limited by flicker problems associated with long refresh intervals.

These drawbacks are not present in self-refreshing display systems (which use internal character storage); these types of displays are capable of displaying large arrays of characters flicker free, and once the computer has input the characters to the display storage, the displays are refreshed automatically without further demands on central processing capacity. Nevertheless, these display systems prove to be of limited utility in many contingent situations. The problem is their relatively slow rate for accepting computer-transmitted information specifying display changes; the fastest available transmission rate in a commercially available unit of this type is 9,600 baud, with $1,200-2,400$ baud the typical rate. ${ }^{15}$ Relatively slow rates such as these limit the temporal contingencies which may be imposed between EPS and the change of displays, the limitation becoming more severe with use of increasingly larger displays.

A self-refreshing display system with a sufficiently rapid input transmission rate would, of course, be more 
flexible for use in contingent situations than most commercial systems; the author has not been able to find a display system with these characteristics. However. a recently published electronic design (Bratt, 1972) for converting a television set into a display terminal has promising performance specifications.

\section{Digital vs Analog Signal Filtering}

The implementation of certain temporal contingencies between stimulus and eye position are limited by transduction delays induced by analog filtering operations performed on the EPS before delivery to the computer. Such delays should be minimized, whenever possible, by replacing the analog filtering with appropriate digital filtering techniques. Of particular concern are capacitance-based analog techniques, which often induce appreciable delays into the filtering process. ${ }^{16}$ A simple example of replacing analog with digital filtering techniques was discussed previously in connection with reducing ac artifacts in the EPS.

\section{Software Considerations}

The software considerations discussed previously in relation to noncontingent paradigms are applicable to the contingent situation. There are, however, additional software problems posed by the timing requirements present in many contingent paradigms. The severity of these timing constraints depends in part on the temporal precision with which the system must react to momentary changes in eye position. The more precision desired, the higher must be the EPS sampling rate, and the more efficient must be the COMPRESS software module which analyzes each EPS sample. To support high sampling rates. the COMPRESS software must run as quickly as possible, and hence should be programmed in Assembly language, trading off the use of extra program storage for faster execution times whenever possible.

One component of the software to be optimized is the subroutine which computes the spatial coordinates of the eye position from sampled EPS voltages. This subroutine is used extensively by the COMPRESS module, being called, for example, by the SPEED and THRESH subroutines. If sufficient core memory is available, one rapid means of computing the spatial position is by means of a voltage lookup table, $\mathrm{P}(\mathrm{X}, \mathrm{Y})$ : the $X$ - and $Y$-EPS voltages (suitably scaled) are used directly to determine the table address $\mathrm{P}(\mathrm{X}, \mathrm{Y})$, at which are stored the corresponding eye-position coordinates. The table can be built during calibration by means of suitable interpolation techniques.

If the visual stimuli presented in a contingent paradigm are presented on a nonstorage-type display system, it is often advisable to program the stimulus-refreshing software so that the refresh cycle can be started at a random part of the display. This technique is particularly advantageous when parts of large stimulus arrays near the momentary fixation point must be changed rapidly in response to changes of fixation; in such cases, regions of the stimulus around the momentary fixation point can be updated immediately, before remaining regions of the display, peripheral to the point of fixation, are updated or refreshed. Such software substantially increases the speed with which a display can be changed in response to eye movements.

\section{REFERENCES}

Atkinson, R. C., Holmgren, J. E., \& Juola, J. F. Processing time as influenced by the number of elements in a visual display. Perception \& Psychophysics, 1969, 6, 321-326.

Bratt, $\mathbf{R}$. TV set is display for data terminal. Electronic Design, $1972,20,134-141$.

Cornsweet, T, N. A new technique for the measurement of small eye-movements. Joumal of the Optical Society of America, $1958,48,808-811$.

Egeth. H. E. Parallel versus serial processes in multi-dimensional stimulus discrimination. Perception \& Psychophysics, 1966, 1 , 245-252.

Estes, W. K., \& Taylor, H. A. A detection method and probability model for assessing information processing from brief visual displays. Proceedings of the National Academy of Sciences, 1964, 52, 446-454.

Haber, R. N. (Ed.) Information processing approaches to visual perception. New York: Holt, Rinehart, \& Winston, 1969.

Hochberg, J. Components of literacy: Speculations and exploratory research. In $\mathrm{H}$. Levin and J. Williams (Eds.), Basic studies in reading. New York: Basic Books, 1970.

Kling, J. W., \& Riggs, L. A. (Eds.) Woodworth \& Schlossberg's Experimental psychology. (3rd ed.) New Yodk: Holt, Rinehart, \& Winston, 1971.

Latour, P. L. Visual threshold during eye-movement. Vision Research, 1962, 2, 261-262.

Latour, P. L. Cortical control of eye-movements. Soesterberg: Institute for Perception RVO-TNO, 1966.

McCormack, $P$. D. Monitoring eye-movements during the learning of paired-associate lists. Technical Report No. 20 Human Performance Center, University of Michigan, Ann Arbor, Michigan, 1970.

Mackworth, J. F., \& Mackworth, N. H. Eye fixation reccrded on changing visual scenes by the television eye-marker. Journal of the Optical Society of America, 1958, 48, 439-445.

Mackworth, N. H. A stand camera for line-of-sight recording. Perception \& Psychophysies, 1967, 2, 119-127.

Mackworth, N. H., \& Bruner, J. S. How adults and children search and recognize pictures. Human Development, 1970, 13 , 149-177.

Neisser, $U$. Visual search. Scientific American, 1964, 210. 94-102.

Rashbass, C. New method for recording eye-movements. Journal of the Optical Society of America, 1960, 50, 642-644.

R ashbass, C., \& Westheimer, G. H. Disunctive eye-movements. Journal of Physiology, 1961, 159, 326-338.

Robinson, D. A. The mechanisms of human smooth pursuit eye-movements. Journal of Physiology, 1965, 180, 569-591.

Smith, W. M., \& Warter, P. J., Jr. Eye-movement and stimulus movement: New photoelectric electromechanical system for recording and measuring tracking motions of the eye. Joumal of the Optical Society of America, 1960, 50, 245-250.

Volkmann, F. C., Schick, A. M., \& Riggs, L. A. Time course of visual inhibition during voluntary saccades. Journal of the Optical Society of America, 1962, 52, 571-578.

Yarbus, A. L. Eye-movements and vision. (Trans. B. Haigh) New York: Plenum Press, 1967.

\section{NOTES}

1. Loftus, G. R. Eye fixations and recognition memory for pictures. Cognitive Psychology, 1972, 3, 525-551. (Published after this manuscript was submitted.)

2. We use, for example, a laboratory-modified version of Model SGH-V2 available from Biometrics, Inc., in Cambridge. Massachusetts.

3. The spontaneous tremor of the eyes, the slow drift, and the small corrective flicks of the eyes (Yarbus, 1967) are not detectable by ocular reflectance techniques.

4. It is possible, but nevertheless very difficult, to measure simultaneously the orientation of the head in space: such information could be combined with ocular reflectance data to estimate eye position in free-moving apparatus.

5. It is important to make each S's biteboard in such a way that movement of the head is minimized while on the biteboard. 
We make deep impression moldings of the mouth by using two to three slabs of dental wax (Kerr impression compound), which are fitted onto a 3/16-in. aluminum plate biteboard.

6. It would be possible to record (in noncontingent situations) the EPS off-line on a multichannel FM instrumentation tape recorder and subsequently play the signals back to the computer through A-D channels for analysis. One FM channel would be needed for each EPS input, and several channels would be needed for recording event markers, calibration pulses, and so forth.

7. The assistance of David H. VanDercar in designing and building the biteboard holder and attached $\mathrm{XYZ}$ movement is gratefully acknowledged.

8. The success of the biteboard apparatus in restricting head movements is readily determined by requesting the $S$ to at tempt head movements while maintaining a fixation point and observing correlated changes in the EPS

9. This will be the case when the tape (or disk) control utilizes a cycle-stealing data-break facility.

10. If the sampling rate is so high that more than $N$ words will be output in an interval during which a single physical black is output onto the mass storage device, more than $2 \mathrm{~N}$ words of buffer storage will be required. In this case, the necessary modifications of RECORD are straight forward

11. These can be averaged or otherwise smonthed over the sequence of samples taken during the fixation interval.

12. The thresholds are most readily determined in units of necessary increase or decrease in $\mathrm{X}$ - and $\mathrm{Y}$-eye-position voltages.

13. The instantaneous velocity is estimated by comparing two or more successive EPS samples in terms of angular displacement information from the calibration matrix.

14. The speed at which displays can be changed also depends on persistence characteristics of the phosphors used. In our display, low-persistence P.31 phosphors are used. Once again, however, the same tradeoff appears: low-persistence phosphors must be refreshed more frequently to avoid flicker in the display.

15. If a standard 8-bit ASCII code is used to represent characters, even a 9,600-baud rate permits a maximum of 1,200 characters/sec, which limits the rate at which a display can be changed to a little more than one new character every millisecond.

16. The Biometrics circuitry (with filter "in"), for example, induces a signal delay of approximately $20 \mathrm{msec}$.

\section{Software character generation*}

\section{CLIFFORD B. GILLMAN and PAUL BUCKLEY \\ University of Wisconsin. Madison. Wisconsin 53706}

Programming considerations are described for computer-controlled visual displays. Software character generation is emphasized.

The cathode ray terminal (CRT) is an excellent general-purpose device for the slay of visual stimuli in psychological research. This paper is concerned with the programming considerations involved in computer-controlled visual displays. Criteria for choice of a CRT have been discussed by Sperling (1971) and Van Gelder (1972). Visual display routines may be used to present strings of alphanumeric characters, text, geometric forms. dot patterns-any visual stimuli. Character generation facilities and display algorithms must be chosen on the basis of the stimuli to be presented. The optimal strategy is ultimately determined by the stimulus material.

The primary decision must be made between hardware and software character generation. Hardware character generators allow very rapid presentation of material, since only a stimulus code must be transmitted from the computer. The character generator is wired to transform this code to the actual stimulus representation. Frequently, additional electronics for automatic refreshment are included, so that central processor involvement is minimized. Speed and automatic refreshment are usually achieved by limiting the stimulus set to the alphanumeric characters. Such CRTs have been called "electronic typewriters." It is impractical, if not impossible, to expand the stimulus set beyond the standard 96 ASCII characters. If this

*This work was supported by Grant MH 19006 from the United States Public Health Service. constraint is acceptable, hardware character generation is optimal. However, generality requires that we discuss the possibility of displaying any visual stimuli that can be drawn, and to do this requires that the character generator be programmable. Software character generation allows maximum flexibility in choice of stimuli at the cost of presenting fewer characters per second. For programmed character generation, a cathode ray oscilloscope (CRO), with suitable interface to a laboratory computer, is necessary.

An additional consideration is the availability of a storage oscilloscope. Nonstorage óscilloscopes require the $E$ to choose a combination of refreshment rate and phosphor decay so that the desired stimulus array is presented without flicker. This commits the central processor to maintaining the visual display. This problem does not exist for storage oscilloscopes, but the storage process produces other potential problems. Erasure of a storage oscilloscope is performed by flooding the entire screen with light, which requires approximately $500 \mathrm{msec}$ to subside. This severely limits the E's ability to present several stimuli in rapid succession and makes dynamic displays impossible. It is also impossible to erase a portion of the display selectively. Although technological developments promise to alleviate these problems while impioving the brightness and resolution of storage oscilloscope displays, generality and flexibility limit this discussion to the standard nonstorage oscilloscope.

The problem is to present and refresh a number of characters as rapidly as possible while maintaining acceptable character detail. However, speed and resolution are but two of many criteria for design of software character generation subroutines. It is also desirable that the $E$ be able to define new stimuli without changing the existing subroutines. The subroutines must be easily called and must occupy as 